\title{
Tratamento Cirúrgico da Síndrome da Apneia Obstrutiva do Sono com Avanço Maxilomandibular: Revisão Sistemática
}

\author{
Surgical Treatment of Obstructive Sleep Apnea Syndrome \\ with Maxillomandibular Advancement: Systematic Review
}

\author{
Rafael de Andrade Balsalobre ${ }^{1}$, Marco Antonio Cardoso Machado ${ }^{2}$, \\ Ligia Maria Juliano ${ }^{2}$, José Fausto de Morais ${ }^{3}$, Luciane Bizari Coin de \\ Carvalho ${ }^{4}$, Lucila Bizari Fernandes do Prado ${ }^{5}$, Gilmar Fernandes do \\ Prado $^{5}$
}

1.Cirurgião-Dentista, Setor de Neuro-Sono do Departamento de Neurologia, Programa de Medicina Translacional do Departamento de Medicine, Universidade Federal de São Paulo.

2.Cirurgião-Dentista, Doutor, Setor de Neuro-Sono, Departamento de Neurologia, Universidade Federal de São Paulo.

3.Estaticista, Doutor, Faculdade de Matemática, Universidade Federal de Uberlândia.

4.Psicóloga, Doutora, Setor de Neuro-Sono, Departamento de Neurologia, Universidade Federal de São Paulo.

5.Médico, doutor, Setor de Neuro-Sono, Departamento de Neurologia, Universidade Federal de São Paulo.

\begin{abstract}
Resumo
Introdução. A cirurgia de avanço maxilomandibular (AMM) tem sido indicada no tratamento da síndrome da apneia obstrutiva do sono (SAOS) em pacientes com apneia grave e deficiência maxilomandibular ou naqueles que não se encaixaram ou não apresentaram melhora com outras modalidades de tratamento. Objetivo. Esta revisão sistemática teve como objetivo avaliar a eficácia da cirurgia de AMM como uma única intervenção para a SAOS em pacientes sem histórico de qualquer procedimento cirúrgico diferente do AMM para o tratamento desta condição. Método. Foi realizada uma revisão sistemática da literatura com base na busca das principais bases de dados bibliográficas. Resultados. Após a busca e seleção de estudos, sete artigos foram incluídos: seis séries de casos e um ensaio clínico. Considerando os resultados de cada estudo, duas meta-análises foram realizada, uma avaliando o desfecho primário IDR e IAH, com reduções médias de 43,71 eventos/h e 35,64 eventos/h. Considerando o desfecho secundário, a sonolência diurna excessiva avaliada com a Escala de Sonolência de Epworth $(n=40)$ diminuiu de 13,98 antes da AMM para 6,58 após $(p<0,0001)$. Conclusão. Esta revisão sistemática sugere que o AMM é um tratamento eficaz para a SAOS, se as devidas indicações forem respeitadas. No entanto, mais estudos com níveis mais altos de evidência, maiores tamanhos de amostra e maior tempo de acompanhamento são necessários.
\end{abstract}

Unitermos. Apneia Obstrutiva do sono; Síndrome da apneia do sono; Cirurgia Maxillomandibular; Meta-analise.

\footnotetext{
Abstract

Introduction. Maxillomandibular advancement (MMA) surgery has been indicated in the treatment of obstructive sleep apnea syndrome (OSAS) in patients with severe apnea and maxillomandibular deficiency or in those who did not fit or did not show improvement with other treatment modalities. Objective. This systematic review aimed to assess the efficacy of MMA surgery as a single intervention for OSAS in patients with no history of any surgical procedure other than MMA for treatment of this condition. Method. We conducted a systematic review of the literature based on a search of the main bibliographic databases.
} 
After the search and selection of studies, only seven could be include in the review: six series of cases and a clinical trial. Considering the outcomes of each study, two meta-analysis could be performed evaluating the primary outcome IDR and IAH, with average reduction 43.71/h e 35.64/h respectively. Another considering the secondary outcome, the excessive daytime sleepiness assessed through Epworth Sleepiness Scale $(n=40)$ before (13.98) and after MMA $(6.58 ; p<0.0001)$. This systematic review suggests that MMA is an effective treatment for OSAS, if proper indications are respected. However, further studies with higher levels of evidence, larger sample sizes, and longer follow-up are required.

Keywords. Obstructive sleep apnea; Sleep apnea syndromes; Maxillomandibular; Surgery; Meta-analysis.

Trabalho realizado no Setor Neuro-Sono da Disciplina de Neurologia Clínica, Escola Paulista de Medicina, Universidade Federal de São Paulo - UNIFESP, São Paulo-SP, Brasil.

Conflito de interesse: não Recebido em: $2016 \quad$ Aceito em: 2016

Endereço para correspondência: Rafael A Balsalobre. Av. Agami, 62 apto 83. Moema. CEP 04522000. São Paulo-SP, Brasil. Tel: 5511 2831-6367. Email: rafabalsalobre@hotmail.com

\section{INTRODUÇÃO}

A Síndrome da Apneia Obstrutiva do Sono (SAOS) é caracterizada por recorrentes episódios de obstrução parcial ou total da via aérea superior durante o sono, que resultam em despertares e dessaturação da oxi-hemoglobina ${ }^{1}$.

A SAOS está associada com o aumento do risco de acidentes ou complicações cardiovasculares e cerebrais, com consequente aumento da morbidade e mortalidade quando não tratada ${ }^{2}$. Outras consequências são os déficits cognitivos, diminuição da libido e também aumento do risco de acidentes automobilísticos devido à Sonolência Excessiva Diurna (SED) $)^{3,4}$.

Os principais fatores de risco para SAOS incluem obesidade, gênero masculino, idade avançada e fatores anatômicos que favoreçam a diminuição da luz da via aérea, como por exemplo, tonsilas palatinas volumosas, palato duro atrésico e retrusão maxilomandibular ${ }^{1}$. 
O padrão ouro para o tratamento da SAOS são os aparelhos de pressão aérea positiva $(\mathrm{APAPs})^{5}$, porém, alguns pacientes submetidos a esta modalidade de tratamento não o toleram por um longo período de tempo ${ }^{6}$. Os aparelhos intra-orais são outra modalidade terapêutica, porém indicados para pacientes com SAOS de grau leve a moderado ${ }^{7}$.

Dentre os tratamentos cirúrgicos, o Avanço Maxilomandibular (AMM) é o procedimento reportado com as maiores taxas de sucesso, com exceção apenas da traqueostomia ${ }^{8}$.

O AMM é um procedimento que proporciona um deslocamento anterior do terço médio e inferior da face concomitantemente. Isto promove também um avanço de todo o tecido mole faríngeo aderido à maxila e à mandíbula, localizado desde a velofaringe até a hipofaringe, permitindo um aumento no calibre da via aérea superior como um todo 9 .

Espera-se que $\bigcirc$ AMM permita um resultado permanente, diferentemente dos APAP's, que necessitam ser usados durante todas as noites para serem efetivos. $O$ AMM está indicado em casos de apneia grave, principalmente quando há recusa ou intolerância ao uso de APAP, ou para pacientes que apresentem alterações maxilomandibulares evidentes, independente da gravidade da SAOS $^{10}$. 
As taxas de sucesso do tratamento da SAOS com AMM variam bastante, provavelmente devido à heterogeneidade clínica, envolvendo idade, gravidade da doença, IMC, dentre outros. Uma revisão sistemática avaliou o efeito do AMM no tratamento da SAOS e reportou taxa de cura $(\mathrm{IAH}<5)$ de $43,2 \%{ }^{11}$. Outra revisão sistemática mostrou que o AMM é eficaz no tratamento da SAOS, obtendo resultados polissonográficos próximos aos conseguidos pela terapia com CPAP ${ }^{12}$.

Entretanto, o fator confundidor dessas revisões foi a inclusão de estudos primários nos quais outras intervenções cirúrgicas além do AMM foram realizadas para o tratamento da SAOS. Ou seja, o AMM não foi a única intervenção avaliada, o que demonstra heterogeneidade clínica e introduz vieses que reduzem a confiabilidade das conclusões. Dessa forma, não é possível saber se os resultados encontrados decorreram dos efeitos do AMM ou se foi do conjunto AMM e outras cirurgias realizadas. Assim, o objetivo desta revisão sistemática foi verificar a eficácia do AMM como intervenção única no tratamento da SAOS.

\section{MÉTODO}

Realizou-se uma Revisão Sistemática da Literatura, com o objetivo de identificar os estudos relevantes publicados na literatura científica que avaliassem a eficácia do AMM como intervenção única no tratamento da SAOS. 


\section{Identificação dos estudos}

Os estudos foram localizados através de estratégias de busca realizadas nas fontes de informações eletrônicas, como as bases de dados bibliográficas e os seguintes portais de revistas eletrônicas: COCHRANE CENTRAL (issue 1, 2015); MEDLINE (1966 a janeiro de 2015); EMBASE (1980 a janeiro de 2015); LILACS (1982 a janeiro de 2015); BBO (1966 a janeiro de 2015).

Realizamos também buscas manuais nas referências bibliográficas dos estudos recuperados nas buscas eletrônicas. Não houve restrição quanto à língua de publicação. Em caso de dúvidas quanto à metodologia aplicada foram enviados e-mails aos autores dos estudos, questionando-os sobre particularidades do desenho e métodos empregados.

As revisões sistemáticas são primariamente baseadas em ensaios clínicos randomizados, porém, devido à grande carência deste modelo de estudo tendo como intervenção o AMM, expandimos a estratégia de busca para todos os tipos de estudo e combinou apenas os descritores da doença com os descritores da intervenção, sem restrição quanto ao tipo de estudo.

\section{Seleção dos estudos}

A identificação dos estudos foi feita por meio dos títulos e resumos dos artigos, e a extração dos dados foi realizada por dois pesquisadores (RAB e MACM). Foram 
selecionados estudos que avaliaram a eficácia do AMM realizado por meio da Osteotomia Le Fort I na Maxila e Osteotomia Sagital Bilateral dos Ramos Mandibulares (OSBRM) como única intervenção cirúrgica no tratamento da SAOS, ou seja, nenhum outro procedimento cirúrgico poderia ter sido realizado anteriormente ou concomitantemente ao AMM. Foram excluídos estudos compostos por série de casos com $n<10$ pacientes $e$ amostras compostas por pacientes com doenças congênitas ou genéticas.

Estudos que possuíam amostras mistas, em que uma parte dos participantes havia sido submetida ao AMM como procedimento cirúrgico único para o tratamento da SAOS e a outra parte recebido, além do AMM, outros procedimentos cirúrgicos, foram excluídos quando não apresentavam os dados individuais de cada paciente para cada desfecho.

\section{Extração de dados}

A avaliação da qualidade dos estudos incluídos foi realizada por três revisores, prevalecendo sempre o consenso nos casos de discordância de opinião.

Em casos de publicações sequenciais em que uma mesma amostra foi utilizada, apenas um estudo foi selecionado, prevalecendo aquele com maior número de pacientes. 
Quando os dados publicados foram reportados apenas com média e desvio padrão, solicitamos aos autores o envio de dados individuais.

Foram extraídos das amostras dos estudos incluídos, dados demográficos, características clínicas e polissonográficas pré e pós-cirurgia de $\mathrm{AMM}$, bem como informações referentes ao procedimento cirúrgico e suas possíveis complicações.

\section{Análise Estatística}

Para possibilitar a inclusão em uma mesma metanálise de estudos com diferentes tipos de desenhos, uma vez que a estratégia de busca realizada foi aberta a todos os tipos de estudo, as seguintes considerações foram tomadas: em estudos série casos, foram considerados como efeito da intervenção os dados obtidos no pós-operatório de cada desfecho analisado e como controle os dados do préoperatório. Na possibilidade de incluir em uma mesma metanálise estudos série de casos e Ensaios Clínicos Randomizados e Controlados (ECRC), apenas o grupo submetido à intervenção do ECRC foi avaliado. Nesse grupo, foram adotados os mesmos critérios de efeito da intervenção e controle como descrito acima nos estudos série de casos.

Nas metanálises que incluíram estudos série de casos e ensaio clínico (braço da intervenção), foi utilizado test $T$ de Student para comparação do IAH basal médio das 
amostras com a finalidade de verificar se o efeito da randomização tinha impacto sobre os resultados.

De acordo com o tipo de informação disponível nos artigos ou obtida entrando em contato com os autores dos estudos correspondentes, os resultados foram agrupados com base nas alterações em relação à linha de base. A análise dos dados foi realizada no software Cochrane Review Manager (RevMan), versão 4.2.8. A diferença da média ponderada foi usada quando dados contínuos estavam disponíveis nos estudos incluídos, com intervalos de confiança de 95\% (IC). A análise estatística usando um modelo de efeito fixo foi usada para estimar os efeitos da intervenção em comparação com o grupo controle. A heterogeneidade entre os efeitos estimados em diferentes estudos foi analisada pelo teste de inconsistência $\left(\mathrm{I}^{2}\right)$ e assumida nos casos em que $I^{2}>50 \%$. A significância estatística foi considerada quando $\mathrm{p}<0,05$.

\section{Desfechos Avaliados}

Foram avaliadas as variações dos seguintes desfechos pré e pós-AMM:

Desfecho primário:

-Variação do Índice de Distúrbios Respiratórios (IDR) pré e pós-AMM/Índice de Apneia/Hipopneia (IAH).

Devido a ausência de esclarecimento quanto aos critérios utilizados para definir o IDR e o IAH nos estudos, decidimos considerar os índices separadamente nas metanálises. 
Desfechos secundários:

-variação da pontuação na Escala de Sonolência de Epworth (ESE)

-vigilância diurna (teste de tempo de reação)

-parâmetros polissonográficos (saturação da oxihemoglobina $\left(\mathrm{SaO}_{2}\right)$, arquitetura do sono, índice de despertares e índice de microdespertares)

-complicações no pós-operatório

Estudos com heterogeneidade clínica não foram agrupados.

\section{RESULTADOS}

A busca eletrônica recuperou 929 estudos (Figura 1) desses, 67 foram eleitos para serem examinados minuciosamente. Quarenta e nove estudos foram excluídos por abordarem outros procedimentos cirúrgicos realizados anteriormente ou concomitantemente à cirurgia de avanço maxilomandibular para tratamento da SAOS. Outros 11 estudos também foram excluídos por se tratarem de séries de casos com menos de 10 participantes. Sete estudos preenchiam corretamente os critérios de inclusão e assim foram selecionados ${ }^{13-19}$ (Tabela 1 ). 
Características da amostra

Foram incluídos na revisão 7 estudos, sendo 6 séries de casos e 1 um ensaio clínico controlado e randomizado, totalizando 171 pacientes.

Figura 1. Quantidade de estudos encontrados, excluídos e selecionados.

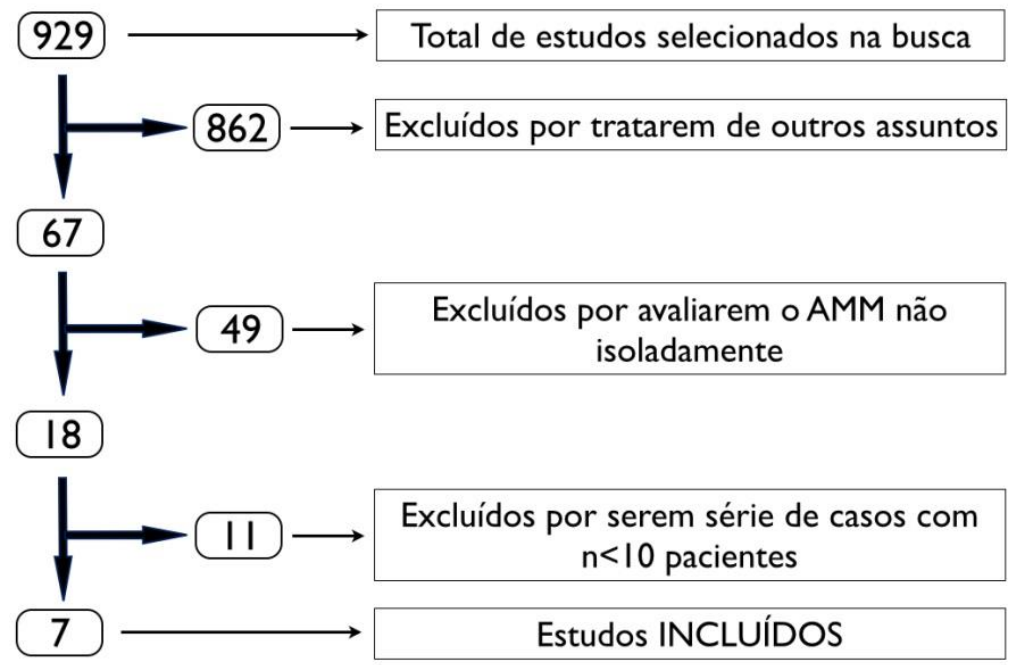

Os estudos de Hochban $1997^{13}$ e Varghese $2012^{18}$ publicaram dados individuais da amostra estudada, o que permitiu descartar casos que haviam sido submetidos a outros procedimentos que não o AMM.

As médias de idade das amostras estudadas variaram entre 41 e 51,5 anos com 88,9\% dos pacientes do gênero masculino. O follow-up médio pós AMM foi de 4,7 meses. Apenas os estudos de Conradt $1998^{14}$ e Vicini $2010^{17}$ relataram o IMC médio dos participantes pré-tratamento, sendo respectivamente $26,7 \pm 2,9$ e $32,7 \pm 5,8 \mathrm{~kg} / \mathrm{m}^{2}$. 
Desfecho Primário: Alterações do IDR/IAH após AMM

Foram formadas duas metanálises avaliando os desfechos primários: alterações do IDR e alterações do $\mathrm{IAH}$.

Tabela 1. Resumo dos estudos incluídos.

\begin{tabular}{|c|c|c|c|c|c|c|c|c|}
\hline Autor / Ano & $\begin{array}{l}\text { Tipo de } \\
\text { Estudo }\end{array}$ & $\mathbf{N}$ & $\begin{array}{l}\text { Índices PSG } \\
\text { Pré Op }\end{array}$ & $\begin{array}{c}\text { Índices PSG Pós } \\
\text { Op }\end{array}$ & Follow-up & $\begin{array}{l}\text { Critério de } \\
\text { Sucesso } \\
\text { Adotado }\end{array}$ & $\begin{array}{l}\text { \% Sucesso } \\
\text { Atingido }\end{array}$ & Desfechos Secundários \\
\hline $\begin{array}{l}\text { Hochban et al., } \\
(1997)^{13}\end{array}$ & $\begin{array}{l}\text { Série de } \\
\text { Casos }\end{array}$ & $36^{*}$ & IAH: $43.52 \pm 15.9$ & $\mathrm{IAH}: 1.86 \pm 2.58$ & 2 meses & $\mathrm{IAH}<10 / \mathrm{h}$ & $97 \%$ & $\begin{array}{c}\text { Avanço Max/Mand: } \\
9.5 / 10.8 \mathrm{~mm}\end{array}$ \\
\hline $\begin{array}{l}\text { Conradt et al, } \\
(1998)^{14}\end{array}$ & $\begin{array}{l}\text { Série de } \\
\text { Casos }\end{array}$ & 24 & IDR: $59.3 \pm 9.6$ & IDR $5.6 \pm 9.6$ & 3 meses & Não considerou & & 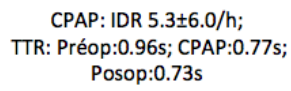 \\
\hline $\begin{array}{l}\text { Gregg et al, } \\
(2000)^{15}\end{array}$ & $\begin{array}{l}\text { Série de } \\
\text { Casos }\end{array}$ & 35 & IDR: $50.5 \pm 15.2$ & IDR: $17.2 \pm 4.8$ & 3-6 meses & $\begin{array}{l}I D R<10 / h \text { ou } \\
\text { redução }>50 \% \text { IDR }\end{array}$ & $\begin{array}{l}89 \% / \\
94 \%\end{array}$ & Avanço Max/Mand: $8 / 10 \mathrm{~mm}$ \\
\hline $\begin{array}{l}\text { Dattilo et al, } \\
(2004)^{16}\end{array}$ & $\begin{array}{l}\text { Série de } \\
\text { Casos }\end{array}$ & 15 & IDR: $76.15 \pm 45.7$ & IDR: $12.59 \pm 12.1$ & $\begin{array}{l}\text { Mín. } 2 \\
\text { meses }\end{array}$ & $\begin{array}{l}I D R<15 / h \text { ou } \\
\text { redução }>50 \% \text { do } \\
\text { IDR }\end{array}$ & $95 \%$ & 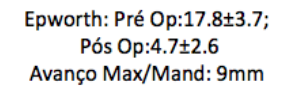 \\
\hline $\begin{array}{l}\text { Vicini et al, } \\
(2010)^{17}\end{array}$ & ECR & 25 & IAH: $57.76 \pm 14.65$ & IAH: $8.16 \pm 6.98$ & 12 meses & Não Considerou & & 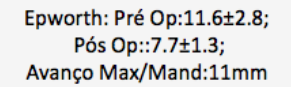 \\
\hline $\begin{array}{l}\text { Varghese et al, } \\
(2012)^{18}\end{array}$ & $\begin{array}{l}\text { Série de } \\
\text { Casos }\end{array}$ & $16^{*}$ & ІАН: $39.0 \pm 19.01$ & IAH: $10.06 \pm 12.2$ & 6.7 meses & $\begin{array}{c}\mid \mathrm{AH}<5 / \mathrm{h} / \\
\mid \mathrm{AH}<10 / \mathrm{h} / \\
50 \% \text { de redução do } \\
\mid \mathrm{AH} \text { e/ou } \mathrm{IAH}<20 / \mathrm{h}\end{array}$ & $\begin{array}{l}31.2 \% / \\
62.5 \% / \\
87.5 \%\end{array}$ & Avanço Max/Mand: 9.7mm \\
\hline $\begin{array}{l}\text { Faria et al, } \\
(2013)^{19}\end{array}$ & $\begin{array}{l}\text { Série de } \\
\text { Casos }\end{array}$ & 20 & IAH: $30.96 \pm 19,24$ & IAH: $10.28 \pm 7,29$ & 6 meses & $\begin{array}{l}50 \% \text { de redução do } \\
\text { IAH basal e/ou } \\
\text { IAH }<20 / \mathrm{h}\end{array}$ & $85 \%$ & Avanço Max/Mand: $6.05 \mathrm{~mm}$ \\
\hline
\end{tabular}

\section{Metanálise 1}

Avaliou as alterações do IDR pré e pós-AMM. Foram incluídos os estudos de Conradt $1998^{14}$, Gregg $2000^{15}$ e Dattilo $2004^{16}$ totalizando 74 pacientes (Figura 2). O gráfico sugere que os resultados obtidos foram favoráveis à intervenção, uma vez que o AMM cirúrgico proporcionou aos pacientes uma redução média de 43,71 eventos/h no IDR $(p<0,00001)$. Com qualidade de evidência muito baixa. 
Figura 2. Variação do IDR pré e pós-AMM.

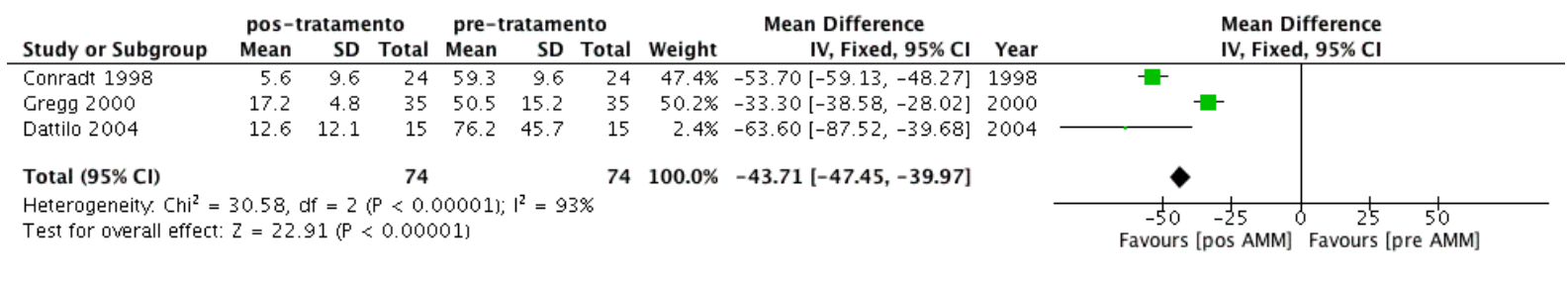

Mean = media; $S D=$ desvio padrão $; \mathrm{WMD}=$ diferença media ponderada $; \mathrm{CI}=$ interval de confiância .

Metanálise 2

Avaliou as alterações do IAH pré e pós-AMM. Foram incluídos os estudos de Hochban $1997^{13}$ e Vicini $2010^{17}$, Varghese $2012^{18}$ e Faria $2013^{19}$ totalizando 97 pacientes (Figura 3). Os resultados obtidos foram favoráveis à intervenção, uma vez que o AMM cirúrgico proporcionou aos pacientes uma redução média de 35,64 eventos/h no IAH $(p<0,00001)$. Com qualidade de evidência muito baixa.

Devido os estudos de Hochban $1997^{13}$, Vicini $2010^{17} \mathrm{e}$ Varghese $2012^{18}$ terem apresentado dados individuais dos pacientes submetidos ao AMM, foi possível constatar que houve uma redução média do IAH pré-AMM de 47,2 $\pm 18,8$

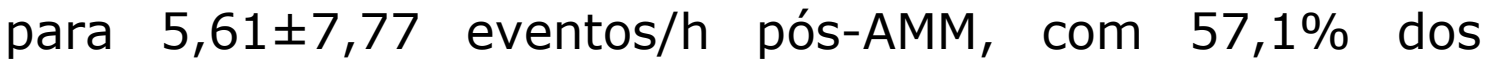
participantes atingindo o critério de cura $(\mathrm{IAH}<5)$. 
Figura 3. Variação do IAH pré e pós AMM.

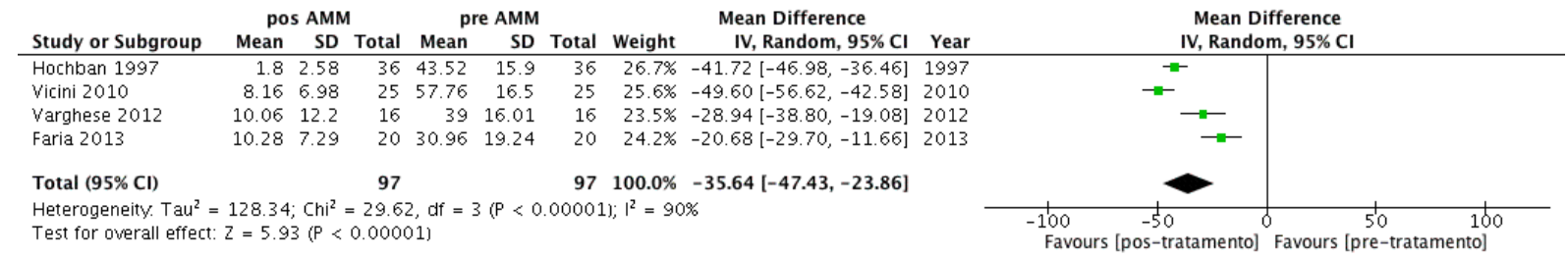

Mean = media; SD = desvio padrão; $\mathrm{WMD}=$ diferença media ponderada; $\mathrm{CI}=$ interval de confiância .

\section{Desfechos Secundários}

\section{Metanálise 3}

Avaliou as alterações da pontuação da ESE pré e pósAMM. Nessa metanálise foram incluídos os estudos de Dattilo $2004^{16}$ e Vicini $2010^{17}$ totalizando 40 pacientes (Figura 4). A intervenção favoreceu uma redução da média da pontuação na ESE de 13,98 pontos para 6,58 após o AMM $(p<0.00001)$. Com qualidade de evidencia muito baixa.

Figura 4. Variação da pontuação da ESE pré e pós AMM.

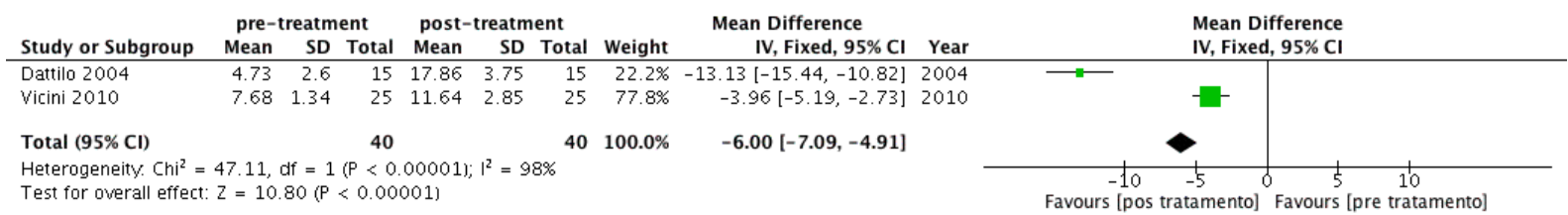

Mean = media; SD = desvio padrão; $\mathrm{WMD}=$ diferença media ponderada; $\mathrm{CI}=$ interval de confiância . 
Metanálise 4

Avaliou as alterações da média das saturações mínimas de $\mathrm{O}_{2}$ pré e pós-AMM. Foram incluídos os estudos de Conradt $1998^{14}$ e Gregg $2000^{15}$ totalizando 59 pacientes (Figura 5). Houve melhora da média de saturação mínima de $\mathrm{O}_{2}$ no pós-AMM. A $\mathrm{SaO}_{2}$ aumentou em média $7,89 \%$ e a saturação média ficou acima de 90\%. Com qualidade de evidência muito baixa.

Figura 5. Variação da saturação mínima de 02 pré e pós AMM.

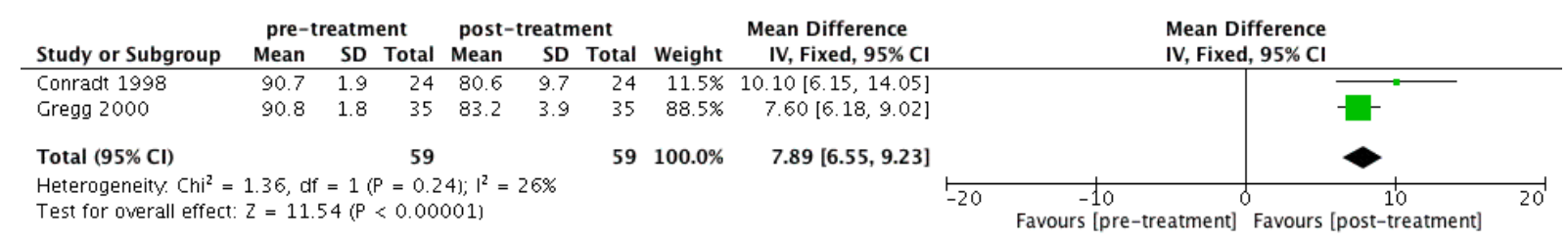

Mean = media $; S D=$ desvio padrão; $\mathrm{WMD}=$ diferença media ponderada $; \mathrm{CI}=$ interval de confiância.

Metanálise 5

Avaliou o impacto da randomização amostral na eficácia da intervenção considerando o desfecho curado $(\mathrm{IAH}<5)$ e não curado $(\mathrm{IAH}>5)$ entre o estudo série de casos de Hochban $1997^{13}$ e o ECR de Vicini $2010^{17}$ (Figura 6). Houve maior eficácia do estudo de Hochban $1997^{13} \mathrm{em}$ relação ao ensaio clínico de Vicini $2010^{17}$. 
Figura 6. Avaliou o impacto da randomização amostral na eficácia da intervenção.

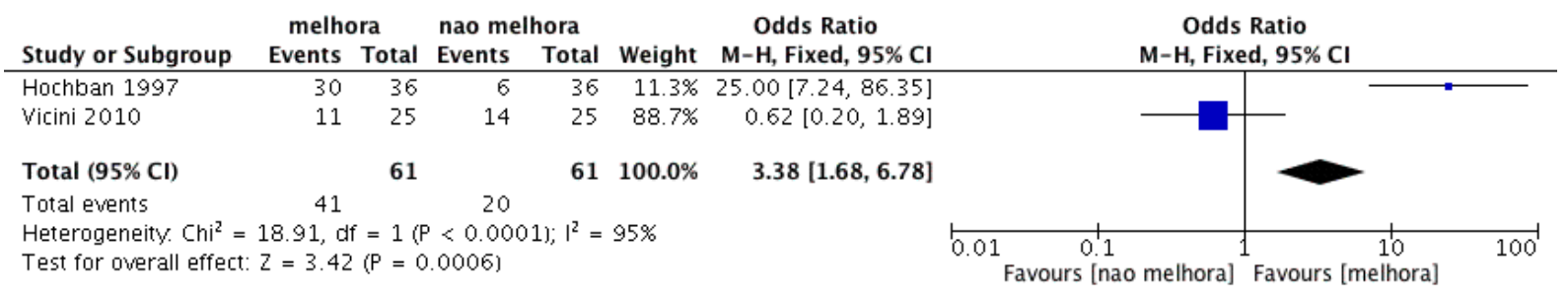

Mean = media; $\mathrm{SD}=$ desvio padrão; $\mathrm{WMD}=$ diferença media ponderada $; \mathrm{CI}=$ interval de confiância .

Outros desfechos avaliados que não permitiram metanálise

O ensaio clínico controlado e randomizado realizado por Vicini $2010^{17}$ comparou a eficácia do tratamento da SAOS com AMM $(n=25)$ e CPAP $(n=25)$ após um ano de follow-up. Ambos os grupos apresentavam homogeneidade pré-intervenção quanto ao $\operatorname{IAH}(p=0,08), \operatorname{ESE}(p=0,08)$, IMC $(p=0,11)$ e idade $(p=0,89)$. As duas modalidades terapêuticas, CPAP e AMM, proporcionaram uma redução tanto do IAH, como na pontuação da ESE; e estas reduções foram semelhantes $(p \geq 0,20)$ em ambos os grupos. A amostra submetida ao AMM mostrou ainda alto grau de satisfação com os resultados obtidos.

Vicini $2010^{17}$ relata que não houve ocorrência de morte durante o intra e pós-operatório. Apenas um caso necessitou de pronto atendimento devido ao entupimento da cânula da traqueostomia. Complicações leves foram mais frequentes, como parestesia no mento e bochecha e pequenas más oclusões corrigidas facilmente ortodonticamente. 
Dattilo $2004^{16}$ compararam os resultados subjetivos (ESE) e objetivos (IDR) obtidos em dois grupos de pacientes apneicos submetidos a fase I $(n=42)$ e a fase II $(n=15)$ do Protocolo de Stanford. Considerando os critérios de sucesso adotados no estudo (IDR $<15$ ou redução $>50 \%$ IDR basal/ESE $<11$ pontos) os pacientes submetidos a fase I e II obtiveram respectivamente $81 \%$ e $100 \%$ de melhora subjetiva (ESE) e $78 \%$ e $93 \%$ de melhora objetiva (IDR).

Conradt $1998^{14}$ avaliou a fragmentação do sono e a vigilância diurna em pacientes apneicos em três situações: basal, com CPAP pré-AMM e pós-AMM. Os resultados demonstraram melhora significante na vigilância diurna com CPAP pré-AMM e pós-AMM quando comparados com os dados basais. $O$ estudo demonstrou ainda diminuição $(p<0,05)$ no índice de microdespertares com CPAP pré-AMM e pós-AMM em relação a situação basal, bem como melhora da arquitetura do sono.

Hochban $1997^{13}$ e Varghese $2012^{18}$ avaliaram em seus estudos, além do IAH outros desfechos secundários, como parâmetros polissonográficos e o ESE. No entanto, estes estudos não puderam ser computados na metanálise, uma vez que os autores forneceram apenas os valores das médias obtidas para todos os pacientes e não os valores individuais dos desfechos avaliados. Sabe-se que em ambos os estudos, alguns pacientes foram submetidos a outros procedimentos cirúrgicos que não o $A M M$, fato este 
que inviabilizou a computação dos resultados no nosso estudo.

Faria $2013^{19}$ avaliou por meio de imagens de Ressonância Magnética o volume da faringe em pacientes apneicos pré e pós-AMM. Os dados obtidos demonstraram o aumento do volume da faringe nas regiões retropalatal e retrolingual após cirurgia de AMM.

\section{DISCUSSÃO}

Como relatado anteriormente, devido à carência na literatura de ensaios clínicos que avaliassem a cirurgia de AMM como procedimento único para o tratamento da SAOS, a estratégia de busca combinou apenas os descritores da doença e da intervenção, tornando-a assim aberta a todos os tipos de estudo. No entanto, mesmo considerando uma estratégia de busca abrangente foi difícil encontrar um número expressivo de estudos que avaliassem o AMM como procedimento único no tratamento da SAOS, uma vez que a maioria deles o avaliou posteriormente ou concomitante a outros procedimentos cirúrgicos.

O baixo nível de evidência científica atribuídos aos estudos primários deve-se ao desenho série de casos e ao " $n$ " reduzido, o que gera grande chance de bias nos resultados obtidos.

Infelizmente, estudos que tiveram parte de sua amostra submetida a outros procedimentos cirúrgicos que 
não o AMM como procedimento único e publicaram apenas a média dos resultados dos valores obtidos nos desfechos avaliados, impossibilitou o uso dos dados em nossa revisão.

Estudos série de casos com $\mathrm{n}<10$ foram excluídos por apresentarem maior chance de viés de seleção e variação de resultados dependentes do volume cirúrgico ${ }^{20}$.

Devido aos satisfatórios resultados demonstrados por revisões sistemáticas recentemente publicadas ${ }^{11,12}$, o AMM cirúrgico tem sido considerado como uma boa opção no tratamento da SAOS, porém essas conclusões estão baseadas em análise de estudos heterogêneos.

Apesar da semelhança com os estudos publicados por Holty e Guilleminalt (2010) ${ }^{11}$ e Pirklbauer et al. $(2011)^{12}$, esta revisão sistemática apresenta um importante cuidado científico, que foi a avaliação do AMM como procedimento cirúrgico único no tratamento da SAOS. Desse modo, o risco de vieses causados pelo impacto de outros procedimentos cirúrgicos que não fosse 0 AMM foi eliminado.

Outra medida importante foi a de incluirmos em metanálises distintas os estudos que consideraram o IAH daqueles que consideraram IDR para avaliar a severidade da SAOS. Dessa maneira, evitamos vieses de super ou subestimação das alterações dos índices de IAH e IDR após AMM, uma vez que o IDR engloba além dos eventos de apneia/hipopneia outros eventos respiratórios. 
As metanálises que avaliaram o desfecho primário (IDR e IAH) apresentaram resultados satisfatórios com redução média dos índices de 43,71 ( $p<0,00001)$ e 35,64 $(p<0,0001)$ respectivamente. Ambas as metanálises foram favoráveis à intervenção, sugerindo que o AMM quando realizado como procedimento cirúrgico único no tratamento da SAOS é eficaz.

A divulgação de dados individuais nos estudos de Hochban $1997^{13}$ e Vicini 2010 ${ }^{17}$, Varghese $2012^{18}$ possibilitou calcular a taxa de cura $(\mathrm{IAH}<5)$ e sucesso (IAH $<20$ e/ou redução $\geq 50 \%$ ) do tratamento, obtendo os valores de 57,1\%, e 90,9\% respectivamente. Essas taxas são superiores aquelas encontradas na revisão realizada por Holty e Guilleminault $(2010)^{11}$, onde obteve taxas de cura e sucesso de $43,2 \%$ e $86 \%$ respectivamente. Uma explicação provável para esse resultado deve-se a acentuada gravidade da SAOS presente nos pacientes incluídos no estudo ${ }^{11}$, com IAH médio basal de 63,9/h. Essa característica da amostra pode justificar o fato de $67 \%$ terem sido submetidos a procedimentos cirúrgicos combinados (AMM + outros procedimentos) na tentativa de se obter sucesso no tratamento.

A carência de ensaios clínicos randomizados em nossa revisão é sabidamente um importante fator de viés em nosso estudo. Essa fragilidade pode ser constatada quando confrontamos as amostras do ECRC de Vicini $2010^{17}$ e a série de casos de Hochban $1997^{13}$, onde, apesar de serem 
homogêneas quanto ao IAH basal $(p=0,0006)$ e a idade $(p=0,011)$, a randomização realizada no ECRC de Vicini $2010^{17}$ pareceu influenciar no resultado final obtido no estudo. A metanálise 5 (Figura 6 ) sugere que os resultados obtidos na amostra não randomizada de Hochban $1997^{13}$ foram mais efetivos que aqueles obtidos na amostra randomizada de Vicini $2010^{17}$.

Quanto aos desfechos secundários avaliados, o AMM foi eficaz na melhora da SED e na normalização da saturação mínima de oxigênio. Com exceção aos riscos inerentes a todo procedimento cirúrgico com anestesia geral, os estudos incluídos não relataram maiores complicações e efeitos colaterais no pós-operatório. Parestesia no mento e bochecha e pequenas más oclusões facilmente corrigidas ortodonticamente foram os efeitos colaterais mais comuns ocorridos nos pacientes submetidos ao AMM (Vicini 2010 ${ }^{17}$ ).

Devido ao curto follow-up apresentado pelos estudos incluídos, que variaram de 2 a 12 meses, pouco pode-se concluir quanto a estabilidade das alterações esqueléticas promovidas pelo AMM, bem como eficiência do tratamento a longo prazo. Estudos que avaliaram a estabilidade e eficiência do AMM a longo prazo encontraram resultados satisfatórios após 12,48 e até 50,7em média de acompanhamento, porém o AMM foi realizado em conjunto com outros procedimentos cirúrgicos ${ }^{21-23}$. No entanto, acreditamos que esses dados possam ser extrapolados para 
o procedimento de AMM quando realizado isoladamente, uma vez que o manejo de estabilização e a quantidade de avanço das bases ósseas são similares.

O estudo de Faria $2013^{19}$ revelou por meio de imagens, o aumento do volume da faringe nas regiões retropalatal e retrolingual, corroborando com os achados de Fairbun (2007) ${ }^{9}$ e Abramson (2011) ${ }^{24}$. No entanto, vale ressaltar as críticas comumente feitas a estudos de imagens de via aérea realizados com pacientes em vigília ou sedados, que, invariavelmente podem não ser representativos do real comportamento dimensional desta durante o sono.

Diferente da revisão publicada por Holty e Guilleminault (2010) ${ }^{11}$ que relacionaram pacientes jovens, baixo IMC, baixo IAH e boa quantidade de avanço maxilomandibular como preditores de sucesso para o tratamento da SAOS com AMM, em nosso estudo, tal associação não pode ser concluída.

Em síntese esta revisão sistemática sugere que os resultados obtidos nos estudos que avaliaram o AMM como procedimento único no tratamento da SAOS são satisfatórios e assemelham-se muito àqueles onde o AMM foi realizado em conjunto a outros procedimentos cirúrgicos.

Porém, vale ressaltar que a decisão em se realizar o AMM isoladamente ou não, depende de fatores como a presença e severidade de alterações anatômicas, a saúde 
geral do paciente bem como seu consentimento e o bom senso do cirurgião em submeter o paciente a várias etapas cirúrgicas na tentativa de ser o mais conservador possível.

\section{CONCLUSÕES}

A cirurgia de Avanço Maxilomandibular como procedimento único ( $\mathrm{AMM}$ ) mostrou-se eficaz no tratamento da Síndrome da Apneia Obstrutiva do Sono (SAOS).

O AMM proporcionou uma redução estatisticamente significante do Índice de Apneia/Hipopneia (IAH) e Índice de Distúrbios Respiratórios (IDR).

O AMM permitiu uma diminuição da pontuação na Escala de Sonolência de Epworth (ESE).

Houve melhora nos níveis de saturação mínima pós AMM.

Apesar dos resultados positivos encontrados no presente estudo, faz-se necessária a realização de estudos com maiores níveis de evidência científica, como os ECRC, em amostras com poder estatístico adequado $e$, principalmente, com longo tempo de acompanhamento.

\section{AGRADECIMENTOS}

Agradecemos a atenção e a ajuda prestada pelo Dr. Walter Hochban e Dra. Ana Célia Faria que nos confiou os dados particulares de seus estudo. 


\section{REFERÊNCIAS}

1.Association ASD. Sleep-related breathing disorders in adults: recommendations for syndrome definition and measurement techniques in clinical research. The Report of Standards of Practice Committee of the American Sleep Disorders Association Task Force Sleep 1999;22:667-89.

2.Collins $R$, Peto $R$, MacMahon $S$, Hebert $P$, Fiebach $N H$, Eberlein $K A$, et al. Blood pressure, stroke, and coronary heart disease. Part 2, Short-term reductions in blood pressure: overview of randomised drug trials in their epidemiological context. Lancet 1990;335:827-38. http://dx.doi.org/10.1016/0140-6736(90)90944-z

3.Beebe DW, Gozal D. Obstructive sleep apnea and the prefrontal cortex: towards a comprehensive model linking nocturnal upper airway obstruction to daytime cognitive and behavioral deficits. J Sleep Res 2002;11:1-16. https://doi.org/10.1046/j.13652869.2002.00289.x

4.Cassel W, Ploch T, Becker C, Dugnus D, Peter JH, von Wichert P. Risk of traffic accidents in patients with sleep-disordered breathing: reduction with nasal CPAP. Eur Respir J 1996;9:2606-11. https://doi.org/10.1183/09031936.96.09122606

5.Sulivan CE, Issa FG, Berthon-Jones M, Eves $L$. Reversal of obstructive sleep apnoea by continuous positive airway pressure applied though the nares. Lancet 1981;1:862-5. https://doi.org/10.1016/s0140-6736(81)92140-1

6. Weaver TE, Grunstein RR. Adherence to continuous positive airway pressure therapy: the challenge to effective treatment. Proc Am Thorac Soc 2008;5:173-8. https://doi.org/10.1513/pats.200708$119 \mathrm{MG}$

7.Kushida CA, Morgenthaler TI, Littner MR, Alessi CA, Bailey D, Coleman J, Jr., et al. Practice parameters for the treatment of snoring and Obstructive Sleep Apnea with oral appliances: an update for 2005. Sleep 2006;29:240-3. https://doi.org/10.1093/sleep/29.2.240 8.Riley RW, Powell N, Li KK, Guilleminault C. Surgical Therapy for Obstructive Sleep Apnea-Hypopnea Syndrome. In: Kryger $\mathrm{MH}$, Roth T, Dement W (eds). Principles and Practice of Sleep Medicine. 3nd ed. Philadelphia: WB Saunders Company, 2000, p.913-28.

9.Fairburn SC, Waite PD, Vilos G, Harding SM, Bernreuter W, Cure $\mathrm{J}$, et al. Three-dimensional changes in upper airways of patients with obstructive sleep apnea following maxillomandibular advancement. J Oral MaxillofacSurg 2007;65:6-12.

https://doi.org/10.1016/j.joms.2005.11.119

10.Prinsell JR. Maxillomandibular advancement surgery for obstructive sleep apnea syndrome. J Am Dent Assoc 2002;133:148997. https://doi.org/10.14219/jada.archive.2002.0079

11. Holty JE, Guilleminault C. Maxillomandibular advancement for the treatment of obstructive sleep apnea: a systematic review and metaanalysis. Sleep Med Rev 2010;14:287-97. 
https://doi.org/10.1016/j.smrv.2009.11.003

12.Pirklbauer K, Russmueller G, Stiebellehner L, Nell C, Sinko K, Millesi $G$, et al. Maxillomandibular advancement for treatment of obstructive sleep apnea syndrome: a systematic review. J Oral Maxillofac Surg 2011;69:e165-76.

13. Hochban W, Conradt R, Brandenburg U, Heitmann J, Peter JH. Surgical maxillofacial treatment of obstructive sleep apnea. Plast Reconstr Surg 1997;99:619-26. https://doi.org/10.1097/00006534199703000-00002

14.Conradt R, Hochban W, Heitmann J, Brandenburg U, Cassel W, Penzel $T$, et al. Sleep fragmentation and daytime vigilance in patients with OSA treated by surgical maxillomandibular advancement compared to CPAP therapy. J Sleep Res 1998;7:217-23.

https://doi.org/10.1046/j.1365-2869.1998.00116.x

15.Gregg JM, Zedalis D, Howard CW, Boyle RP, Prussin AJ. Surgical alternatives for treatment of obstructive sleep apnoea: review and case series. Ann R Australas Coll Dent Surg 2000;15:181-4.

16.Dattilo DJ, Drooger SA. Outcome assessment of patients undergoing maxillofacial procedures for the treatment of sleep apnea: comparison of subjective and objective results. J Oral Maxillofac Surg 2004;62:164-8. https://doi.org/10.1016/j.joms.2003.03.002

17.Vicini C, Dallan I, Campanini A, De Vito A, Barbanti F, Giorgiomarrano $\mathrm{G}$, et al. Surgery vs ventilation in adult severe obstructive sleep apnea syndrome. Am J Otolaryngol 2010;31:14-20. https://doi.org/10.1016/j.amjoto.2008.09.002

18. Varghese R, Adams NG, Slocumb NL, Viozzi CF, Ramar K, Olson EJ. Maxillomandibular advancement in the management of obstructive sleep apnea. Int J Otolaryngol 2012;2012:373025. https://doi.org/10.1155/2012/373025

19.Faria AC, da Silva-Junior SN, Garcia LV, dos Santos AC, Fernandes $M R$, de Mello-Filho FV. Volumetric analysis of the pharynx in patients with obstructive sleep apnea (OSA) treated with maxillomandibular advancement (MMA). Sleep Breath 2013;17:395-401. https://doi.org/10.1007/s11325-012-0707-1

20.Capobianco DM, Batilana A, Gandhi M, Shah J, Ferreira R, Carvalho $E$, et al. Surgical treatment of sleep apnea: association between surgeon/hospital volume with outcomes. Laryngoscope 2014;124:320-8. https://doi.org/10.1002/lary.24207

21. Miles PG, Nimkarn Y. Maxillomandibular advancement surgery in patients with obstructive sleep apnea: mandibular morphology and stability. Int J Adult Orthodon Orthognath Surg 1995;10:193-200.

22. Nimkarn Y, Miles PG, Waite PD. Maxillomandibular advancement surgery in obstructive sleep apnea syndrome patients: long-term surgical stability. J Oral Maxillofac Surg 1995;53:1414-8. https://doi.org/10.1016/0278-2391(95)90667-3

23.Li KK, Powell NB, Riley RW, Troell RJ, Guilleminault C. Long-Term Results of Maxillomandibular Advancement Surgery. Sleep Breath 2000;4:137-9. https://doi.org/10.1007/s11325-000-0137-3 
24.Abramson Z, Susarla SM, Lawler M, Bouchard C, Troulis M, Kaban LB. Three-dimensional computed tomographic airway analysis of patients with obstructive sleep apnea treated by maxillomandibular advancement. J Oral Maxillofac Surg 2011;69:677-86. https://doi.org/10.1016/j.joms.2010.11.037 